\title{
On the 3-dimensional structure of the streamer belt of the solar corona
}

\author{
F. Saez ${ }^{1}$, A. N. Zhukov ${ }^{2,3}$, P. Lamy ${ }^{1}$, and A. Llebaria ${ }^{1}$ \\ 1 Laboratoire d'Astronomie Spatiale (CNRS) Les Trois Lucs, BP 8, 13376 Marseille Cedex 12, France \\ e-mail: fabien.saez@oamp.fr \\ 2 Royal Observatory of Belgium, Avenue Circulaire 3, 1180 Brussels, Belgium \\ ${ }^{3}$ Skobeltsyn Institute of Nuclear Physics, Moscow State University, 119992 Moscow, Russia
}

Received 15 September 2004 / Accepted 5 July 2005

\section{ABSTRACT}

We investigate the three-dimensional structure of the streamer belt of the solar corona using a model that allows us to simulate its quasistationary configuration. Starting from the National Solar Observatory photospheric magnetograms, the position of the neutral line at the source surface $\left(2.5 R_{\odot}\right)$ is determined using the potential field source surface model. A plasma sheet is centered around the current sheet represented as the radial extension of the neutral line. Comparing the synoptic maps of the streamer belt obtained with the SOHO/LASCO-C2 coronagraph and the simulated synoptic maps constructed from our model of the warped plasma sheet, we confirm earlier findings by Wang et al. (1997, ApJ, 485, 875; Wang et al. 2000, Geophys. Res. Lett., 27, 149) that the streamers are associated with folds in the plasma sheet. Although the large-scale structure of the streamer belt is described reasonably well, some features, however, cannot be explained in this framework. We propose that two types of large-scale structures take part in the formation of these additional features. The first one is an additional fold of the neutral line, which does not appear in the modeled source surface neutral line, but is well visible in photospheric magnetograms. The second one is a plasma sheet with a ramification in the form of a secondary short plasma sheet. We show that these structures better describe the observed configurations of the streamer belt. The secondary plasma sheet can be formed between two secondary current sheets connected to the main current sheet. Our results suggest that the potential field source surface model is not fully adequate for the description of the fine structure of the streamer belt, even during the time of low solar activity.

Key words. Sun: corona - Sun: magnetic fields

\section{Introduction}

The large-scale structure of the solar corona is dominated by the coronal holes and helmet streamers. During the years of low solar activity, the coronal holes occupy the polar regions and the streamers are concentrated near the solar equatorial plane (streamer belt). When the activity increases, the polar coronal holes shrink and the streamer belt widens as the streamers appear also at higher latitudes. During the maximum of activity, the polar coronal holes disappear (although small coronal holes may still occur, also at low latitudes), and the corona is dominated by streamers all around the solar disk. This picture is now well established using the observations of the corona during total solar eclipses (see e.g., Vsekhsvyatsky et al. 1965) and by coronagraphs. In particular, the Large Angle Spectroscopic Coronagraph (LASCO, see Brueckner et al. 1995) onboard the Solar and Heliospheric Observatory (SOHO) provided detailed information on the evolution of the structure of the corona during the current solar cycle.

Attempts to associate the streamer belt, the source surface neutral line (derived from potential extrapolations of the photospheric magnetic field) and the heliospheric current sheet have been undertaken for a long time (e.g. Schatten 1971; Pneuman \& Kopp 1971; Howard \& Koomen 1974; Wang et al. 1997;
Koomen et al. 1998; Wang et al. 2000; Smith 2001). Wang et al. (1997) showed that the structure of the streamer belt observed during the low-activity years originates from a single tilted and warped plasma sheet centered around the neutral current sheet. When the edge-on parts of the plasma sheet rotate in and out of the plane of the sky, they produce arc-shaped features which are very common in the LASCO synoptic maps. In general, the streamer belt configuration can well be described in this way (Wang et al. 2000), especially its large-scale features, including the east - west asymmetry produced partly by the tilt of the solar rotation axis to the plane of the sky. At larger heliospheric distances this current sheet is observed in situ as the heliospheric current sheet (HCS). When the Sun rotates, it produces the well-known sector structure of the interplanetary magnetic field (e.g. Howard \& Koomen 1974). Other authors (Molodensky et al. 1996; Vedenov et al. 2000) consider that even the lower part of a streamer (its helmet) results from a pleat in the HCS.

Some fine features of the LASCO synoptic maps remain without their counterparts in the synoptic maps constructed using the model of Wang et al. (2000). They are also absent in the simulations using 3D magnetohydrodynamic (MHD) models (e.g., Linker et al. 1999). These models make no 
assumptions on the distribution of coronal currents (e.g. the presence of a current sheet), and only describe the general configuration of the streamer belt. Liewer et al. (2001) proposed that additional fine streamers result from flux tubes containing plasma of higher density and not from folds in the plasma sheet: these narrow high-density bright rays naturally produce the fine streamer-like structures near the main streamer belt. Using polarization analysis, Koutchmy (1972) and Badalyan et al. (1997) found that the line-of-sight dimension of the streamer stalk is close to its width in the plane of the sky; the streamer thus resembles a ray rather than a sheet. Eselevich \& Eselevich (1999) proposed that the whole streamer belt consists of fine bright rays, though citing only the visual appearance without a detailed investigation of the three-dimensional structure of the belt.

In this paper we propose another interpretation of the 3D structure of the streamer belt that allows us to explain the existence of fine features in the LASCO synoptic maps by additional folds and/or ramifications of the main plasma sheet.

\section{The method}

We follow the method proposed by Wang et al. (1997) which consists in comparing synoptic maps of the radiance of the solar corona constructed from LASCO-C2 images with modeled synoptic maps created by calculating the Thomson scattering of an assumed 3D distribution of electrons. The LASCO$\mathrm{C} 2$ images ( $>50$ per day) are first corrected for instrumental effects and then calibrated in units of solar radiance. Our pipeline processing essentially includes correcting the dates of observations and the exposure times, restoring the missing zones of $32 \times 32$ pixels resulting from telemetry dropouts, devignetting, and subtracting the F-corona and the instrumental straylight (the relevant images are generated on a daily basis from the routine polarization sequences and then interpolated to the time of observation). The resulting images of the radiance of the K-corona are resampled to the common format of $512 \times 512$ pixels which is adequate for generating synoptic maps.

The synoptic maps used in this work differ from standard synoptic maps where the east and west limbs are separated. As first introduced by Lamy et al. (2002), the full circular profiles $\left(360^{\circ}\right)$ of the solar corona extracted from LASCO-C2 images in the counterclockwise direction starting at south, are stacked as a function of time, which goes from left to right, offering a global view of the solar corona. In this work, these synoptic maps are reversed in such a way that the longitude increases from left to right to allow a direct comparison with magnetograms so oriented. The process of building the synoptic maps starts with a transformation from the standard Cartesian coordinates of the images ( $X$ and $Y$ ) to polar coordinates, i.e., position angle (horizontal or $X$-axis) and radial distance (vertical or $Y$-axis). The center is naturally the center of the solar disk which is accurately determined from the positions of the stars present in the images. In this article, we display the profiles at $2.5 R_{\odot}$ with an angular step of $1^{\circ}$. A uniform resampling at 1000 samples per Carrington rotation (37 samples per day) is applied to remove the effect of irregular image acquisition.

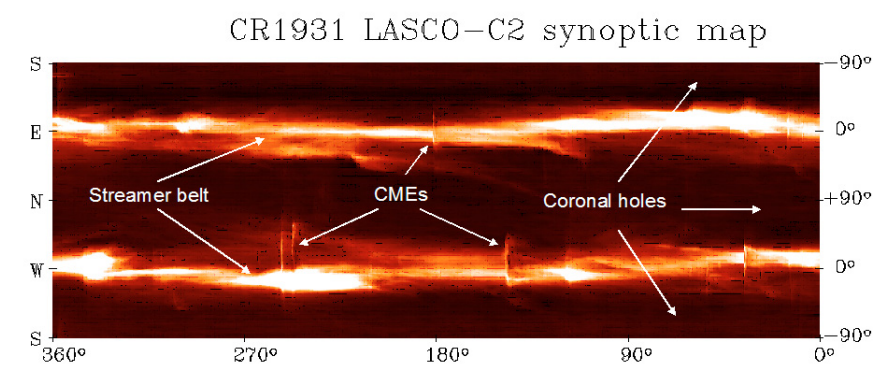

Fig. 1. An example of a non-reversed LASCO-C2 synoptic map at $2.5 R_{\odot}$ corresponding to the Carrington rotation 1931 .

These synoptic maps of the K-corona, calibrated in units of mean solar brightness are thus fully quantitative.

Figure 1 displays the synoptic map corresponding to Carrington Rotation (CR) 1931 as an example. The main features as indicated are clearly distinguished because of their different signatures. The streamer belt is globally continuous but does exhibit several substructures. The coronal mass ejections (CME) are transient phenomena with short lifetimes (up to several hours) and they appear as vertical streaks roughly superimposed on the belt. Note the large extension of the north and south coronal holes.

Starting from the National Solar Observatory ${ }^{1}$ photospheric magnetograms, the position of the neutral line at the source surface $\left(2.5 R_{\odot}\right)$ is determined using a source surface model with the potential approximation. The neutral sheet is defined by the radial extension of the neutral line and the plasma sheet is centered around the neutral sheet. Assuming a Gaussian-like distribution of electron density as a function of distance to the neutral sheet, we are able to build a full 3D model of the electron density distribution in the outer corona ( 2.5 to $6.5 R_{\odot}$ ). The details of the model will be given in a forthcoming paper (Saez et al. 2005). To be able to manage such a large volume of data, we use the adaptative mesh method called octree (Vibert et al. 1997). The 3D density distribution data cube is projected onto images of $512 \times 512$ pixels taking into account the Thomson scattering. The modeled synoptic maps are constructed like those from the LASCO images. Such a method further allows a quantitative comparison.

\section{Results}

For the purpose of the present analysis, we consider the case of the Carrington rotations (CR) 1911 and 1913. They respectively correspond to the periods of June 28-July 25, 1996 and August 22-September 18, 1996, when the solar activity was very close to its minimum during cycle 23 . Accordingly, we expect that the configuration of the outer corona will be entirely dominated by the streamer belt associated with the neutral sheet. Figure 2a displays the neutral line calculated from the NSO magnetograms. The $Y$-axis corresponds to latitudes as in the LASCO synoptic maps. The $X$-axis represents longitudes increasing from left to right. The most pronounced feature is a large undulation over the longitude range $180^{\circ}-300^{\circ}$

1 The NSO web site: http://www.nso.edu/ 

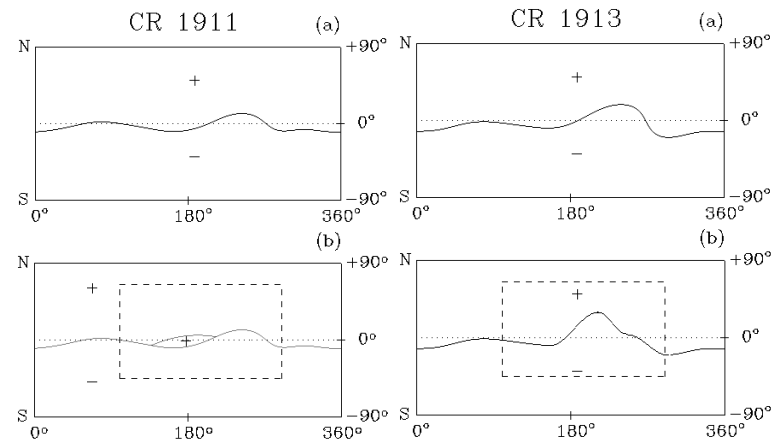

(c)

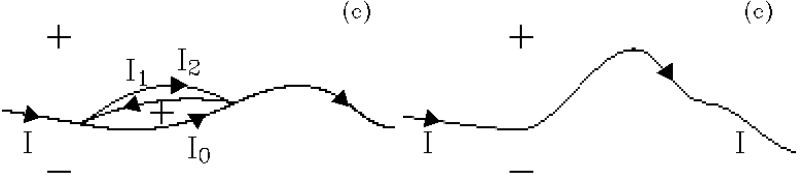

Fig. 2. Configurations of the plasma sheet. "Plus" and "minus" mark the regions of positive and negative magnetic field polarity. a) The neutral sheet computed from NSO magnetograms for CR 1911 (left) and CR 1913 (right). b) The configuration with an additional secondary plasma sheet for CR 1911 (left), and with an additional fold for CR 1913 (right). c) Enlargements of the plasma sheet with the currents crossing the current sheets. Current directions are shown by arrows.

which persists but evolves from CR 1911 to CR 1916 showing an increasing amplitude.

Figures $3 \mathrm{a}$ and $\mathrm{b}$ display the LASCO-C2 and the calculated synoptic maps of the $\mathrm{K}$-corona radiance at $2.5 R_{\odot}$ for both periods. We did not attempt to accurately match the width of the belt and purposely made it thinner in our model to delineate the various features. The modeled structures are altogether in good agreement with those seen on the LASCO-C2 maps, thus confirming earlier findings by Wang et al. (2000) that the streamers can be represented as folds in the plasma sheet. The most notable discrepancy can be seen at the western limb in the longitude ranges $135^{\circ}-225^{\circ}$ for the CR 1911 and $225^{\circ}-290^{\circ}$ for the CR 1913 . The LASCO maps (Fig. 3a) show three arc-shaped structures marked by arrows (they are more clearly seen in CR 1913) whereas the modeled maps (Fig. 3b) exhibit only two. We note that the 3D MHD model performed for the CR 1913 by Linker et al. (1999) led to a configuration with only two arc-shaped features. The additional arcs seen in the LASCO images are strikingly similar to those resulting from the warped plasma sheet. We therefore hypothesized that these arcs result from plasma sheets that do not correspond to the source surface neutral line. The modified configurations of the plasma sheet that appear to better match the additional arcs on the synoptic maps are sketched in Fig. 2b. Indeed, the improved model is able to better reproduce the observations as three arcs now appear in Fig. 3c as indicated by the arrows. For the CR 1913, we kept the single plasma sheet configuration, only introducing an additional fold around longitude $210^{\circ}$ (Fig. 2b). For the CR 1911, we however introduced a secondary plasma sheet in the longitude range $150^{\circ}-210^{\circ}$.

Let us now elucidate these modified configurations of the plasma sheet, their magnetic structure and connections with the photospheric field by inspecting the synoptic maps of the photospheric magnetic field for the relevant Carrington rotations. They are displayed in Fig. 4 where we have further superimposed the large scale photospheric neutral lines. These lines have been obtained by first applying a $3 \times 3$ pixel box average, then by setting the positive pixels to one and the negative pixels to zero, and finally drawing the boundaries between the two domains. For the CR 1913 (Fig. 4b), the photospheric neutral line exhibits three horizontal branches at latitudes of $\sim 60^{\circ}$ north, $\sim 0^{\circ}$ and $\sim-60^{\circ}$ south, in the longitude range $225^{\circ}-290^{\circ}$. In the same range, they correspond to three streamers seen in the LASCO synoptic map (Fig. 3a). To confirm this correspondence, we inspected EIT (Extreme-ultraviolet Imaging Telescope, see Delaboudinière et al. 1995) and LASCO images around times when the three streamers had the lowest apparent latitude. These times can be determined from the LASCO synoptic maps with an accuracy of about 2 days and correspond to the times when the respective parts of the plasma sheet are nearly perpendicular to the plane of the sky. At these times, we expect to see, in the plane of the sky, a system of loops below each streamer as illustrated in the model of Pneuman \& Kopp (1971, see their Fig. 3). These loops overlie the photospheric neutral line stretched in the east-west direction.

We then followed the method used by Schwenn et al. (1997) who investigated the connection of LASCO C1 and C2 streamers with photospheric magnetograms. Figure 5 shows three combined EIT and LASCO C2 images corresponding to times when the streamers 1, 2 and 3 (see Fig. 3a) have the lowest apparent latitudes, i.e. when the respective parts of the plasma sheet are perpendicular to the plane of the sky. As expected, three systems of loops 1,2 and 3 are located below the streamers. In turn, the loop systems overlie the respective photospheric neutral lines when they reach the west limb. For example, streamer 2 is located above the large-scale loops which are most clearly visible in the EIT Fe XIV (284 ̊) bandpass on September 4 (Fig. 5a). This structure had passed the central meridian 7 days earlier, i.e., around August 28, so the neutral line under these loops should be located at longitude $\sim 270^{\circ}$. Indeed, Fig. $4 \mathrm{~b}$ shows a portion of the neutral line close to the equator at longitude $260^{\circ}$, which is stretched in the east-west direction. Streamers 3 and 1 correspond to large-scale loops best seen at the west limb on September 4 and 6 respectively (Figs. 5b, c). In the same way, we determined their correspondence to photospheric neutral lines at $\sim 270^{\circ}, \sim 60^{\circ}$ north, and $\sim 280^{\circ}, \sim 60^{\circ}$ south (Fig. 4 b). The correspondence of the structures observed by EIT and LASCO is not radial as the solar wind flow from the polar coronal holes makes the streamer belt flatter with increasing latitude. Note also that the "vertical" part of the neutral line in the active region located at the west limb on September $4\left(\sim 260^{\circ}, \sim 15^{\circ}\right.$ south, on the photosphere, see Fig. 4b), corresponds to the diffuse structure seen in Fig. 3a (right column) between the streamers 2 and 3, i.e., to the current sheet close to the plane of the sky. The loops are not visible in this case as they are nearly perpendicular to this plane.

The situation for the CR 1911 is different. The photospheric magnetic field synoptic map shown in Fig. 4a does not exhibit any additional large-scale folds of the photospheric neutral line in the northern hemisphere at longitude $\sim 180^{\circ}$. 

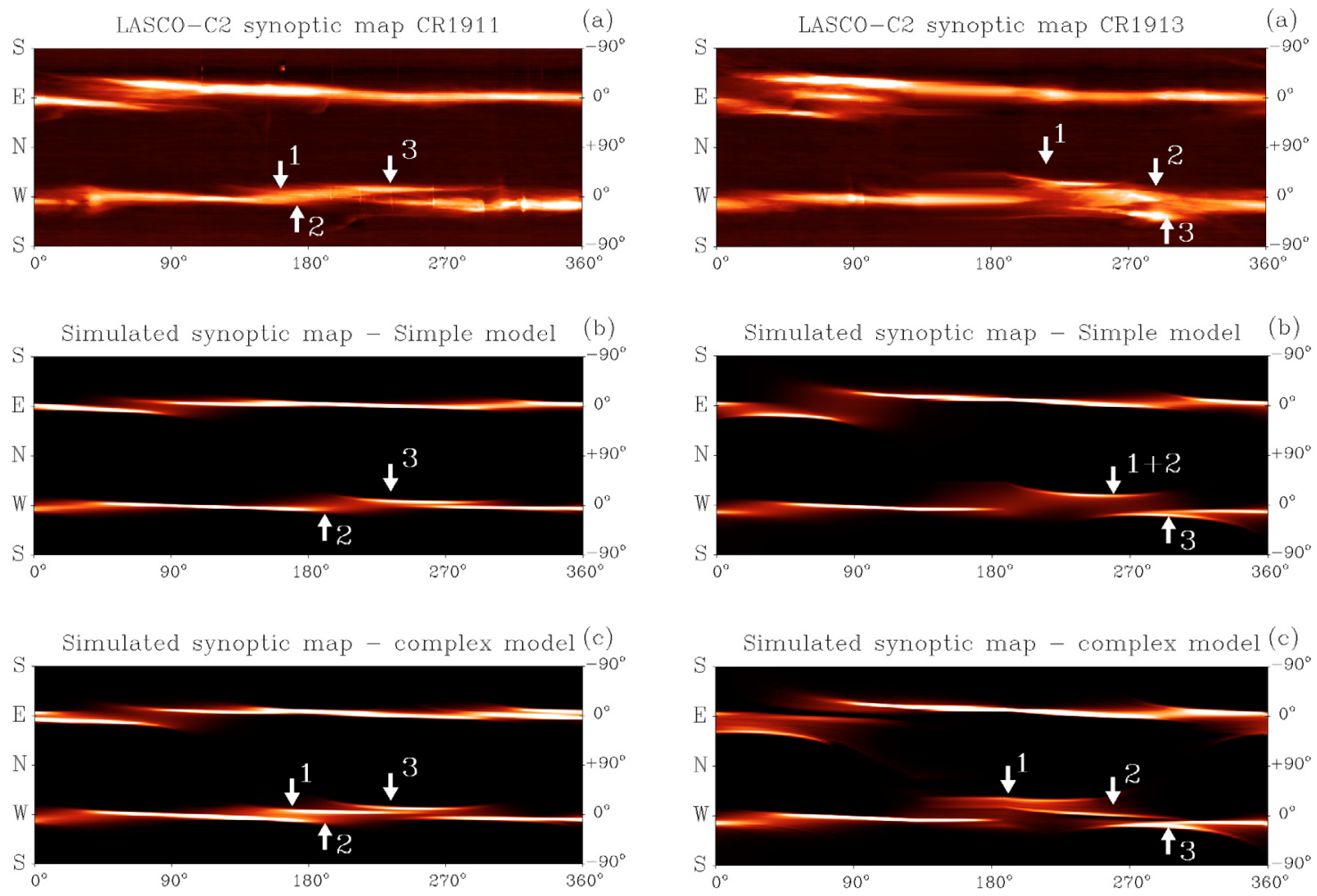

Fig. 3. Synoptic maps for the CR 1911 (left column) and CR 1913 (right column) constructed from: a) LASCO-C2 images taken during the corresponding Carrington rotation period; b) simulated images using the single plasma sheet configuration, only displaying two streamers, highlighted by two arrows; c) simulated images using both the main and the secondary plasma sheets, where three structures are present, indicated by three arrows. The three arrows mark the fine arc-shaped structures under consideration (see text).

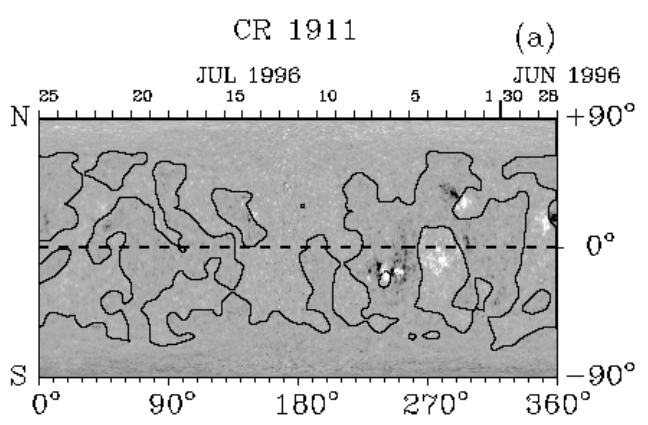

CR 1913

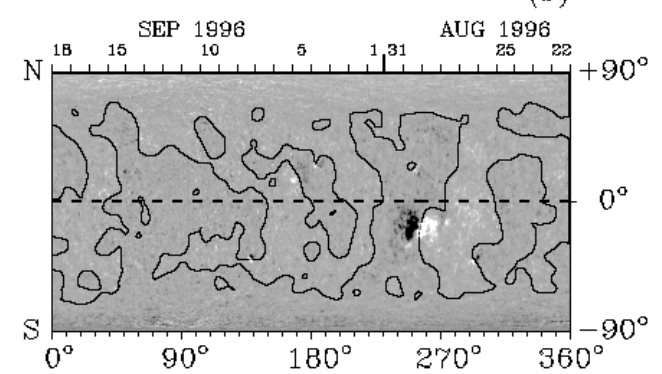

Fig. 4. Photospheric magnetic field synoptic maps for the CR 1911 (a) and CR 1913 (b). Dates on top refer to the times when the features at the corresponding Carrington longitude (lower scale) pass the central meridian. The white (resp. black) color indicates the positive (resp. negative) line-of-sight component of the photospheric magnetic field. The superimposed solid lines represent the large-scale photospheric neutral lines separating positive and negative large-scale fields.
We performed similar comparisons of EIT and LASCO images (Fig. 6) with photospheric magnetograms (Fig. 4a). Streamer 3 (Fig. 3a) is close to the plane of the sky (having the lowest apparent latitude) on July 13-14. Figure 6a shows the images taken when this streamer is clearly separated from the equatorial streamer. The loops below streamer 3 are clearly seen in the EIT $284 \AA$ images and overlie the horizontal part of the photospheric neutral line seen in Fig. 4 a at $\sim 280^{\circ}, \sim 45^{\circ}$ north. This streamer is strongly non-radial at low heights. The equatorial streamer seen in Fig. 6a corresponds to the neutral line at $280^{\circ}, 10^{\circ}$ north, linked to an active region. Streamer 2 corresponds to the long (perhaps intermittent) horizontal part of the neutral line in the longitude range $0^{\circ}-210^{\circ}$ and the latitude range $40^{\circ}-45^{\circ}$ south. Figures $6 \mathrm{~b}$ and c show weak loop structures below this streamer, better seen in the Fe XII (195 ̊) EIT bandpass. On the contrary, streamer 1 (in the plane of the sky on $\sim$ July 19 , see Fig. 6 b, and most clearly separated from streamer 2 on July 21, see Fig. 6c) does not correspond to any noticeable horizontal part of photospheric neutral line. The only prominent nearby feature in the northern hemisphere is the small bipole at $150^{\circ}, 15^{\circ}$ north.

However it is about $30^{\circ}$ offset to the east, and its neutral line is short and stretched in the north - south direction, so it cannot correspond to a plasma sheet perpendicular to the plane of the sky. Moreover, this bipole is seen at the central meridian on July 15, and it seems to have decayed at the time when it reached the west limb (July 22) as no distinct features are seen 


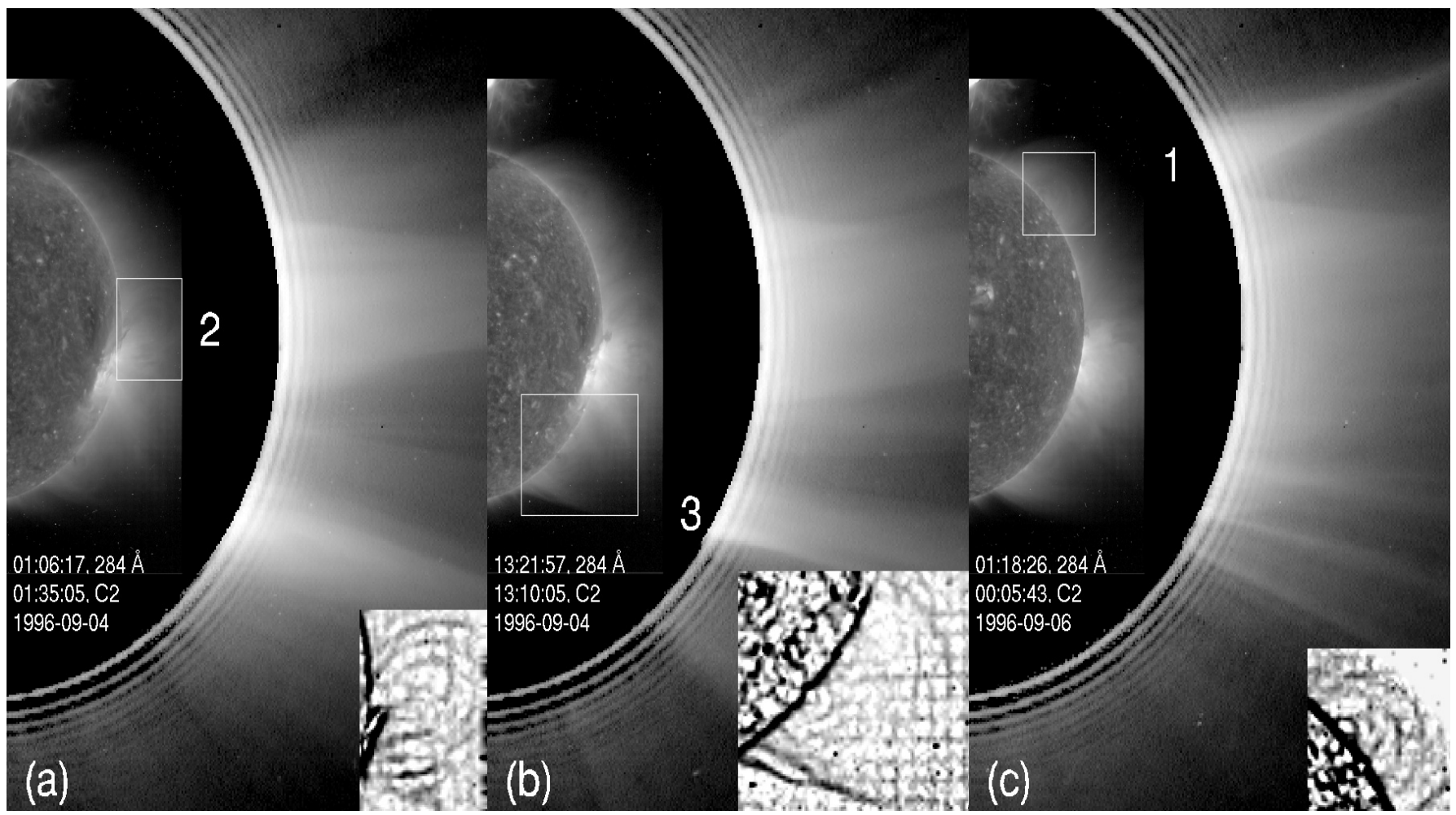

Fig. 5. Combined SOHO/LASCO C2 and SOHO/EIT $284 \AA$ (Fe XIV) bandpass images showing the correspondence of streamers 1,2 and 3 to large-scale loops for the CR 1913. Insets represent boxed areas processed with the "Mad Max" filter (Koutchmy \& Koutchmy 1989) and magnified to better show the loop structures. All times are UT.

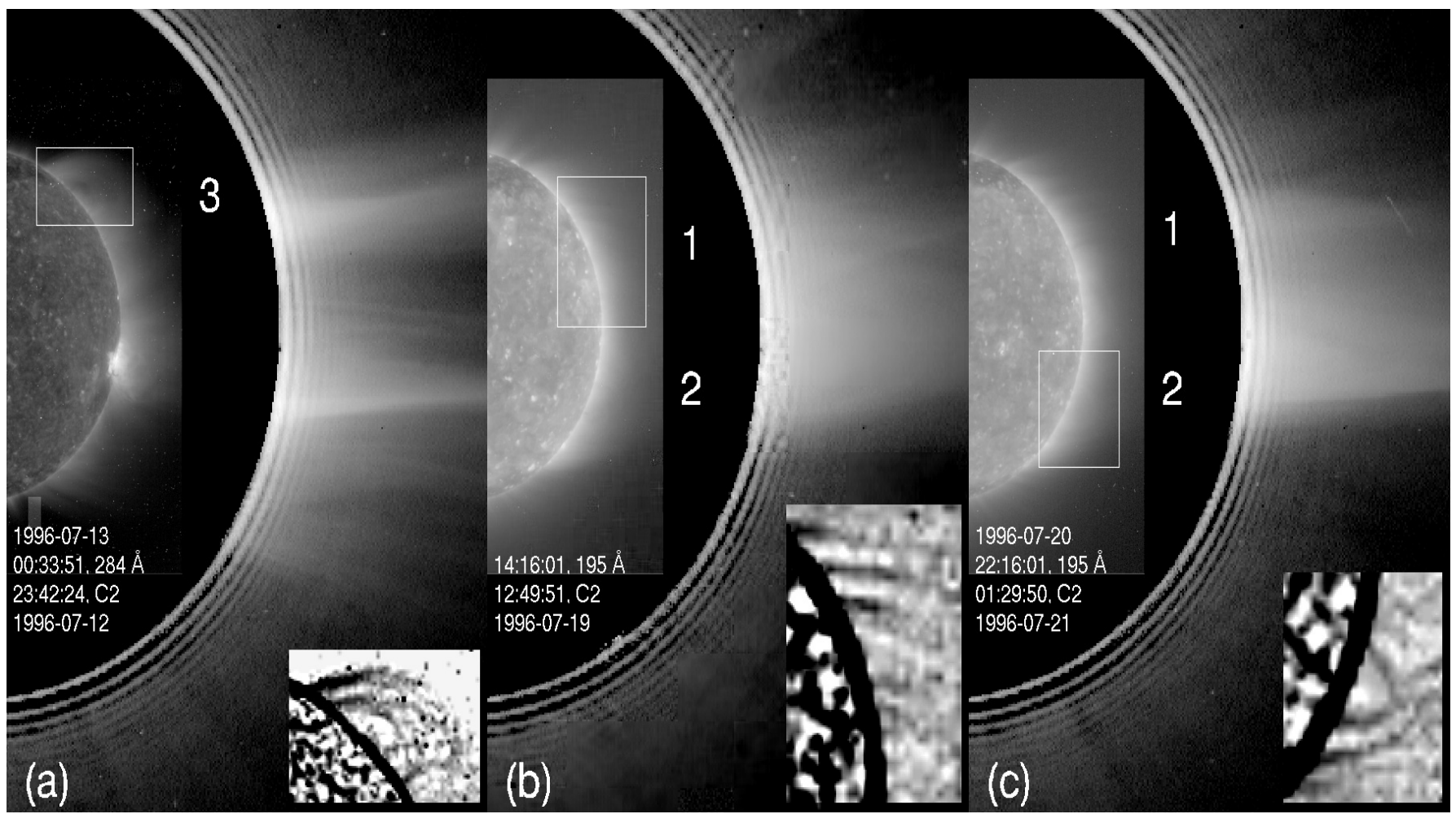

Fig. 6. Combined SOHO/LASCO C2 images and SOHO/EIT images at $284 \AA$ (a) and $195 \AA$ (b, c) showing the correspondence of streamers 2 and 3 to large-scale loops and the lack of such correspondence for the streamer 1 for the CR 1911. Insets represent boxed areas processed with the "Mad Max" filter (Koutchmy \& Koutchmy 1989) and magnified to better show the loop structures. All times are UT. 
above it. So, no loop-like structures are seen under streamer 1 in Figs. $6 \mathrm{~b}$ and c. The investigation of other EIT images taken in the other three bandpasses around this time confirms this conclusion. Therefore, in the case of CR 1911, only streamers 2 and 3 can be described in the "photospheric neutral lineloop system-streamer" framework. Streamer 1 is not related to a photospheric neutral line, so we hypothesized that it corresponds to a plasma sheet unrelated to the neutral sheet (Fig. 2). We note that the arc introduced by this additional plasma sheet on the eastern limb at longitude $\sim 0^{\circ}$ is absent in the LASCO synoptic map (Fig. 3), thus implying that the ramification had disappeared at that time. Even more fine features can be seen nearby (at longitudes $0^{\circ}-90^{\circ}$ ), again suggesting a more complex plasma sheet structure.

\section{Discussion}

We have demonstrated in the previous section that the fine structures of the streamer belt for the CR 1911 can be explained by multiple plasma sheets. We stress here that, for the purpose of our 3D model, these plasma sheets are artificially introduced as sheet-like structures with an enhanced density in comparison to the ambient plasma. Let us now address the magnetic configuration of these plasma sheets in more detail and examine how a multiple (double, in our case) plasma sheet can be maintained.

We have specified so far the magnetic field configuration (neutral current sheet) of the main plasma sheet only. It is illustrated in a simplified plane-parallel $1 \mathrm{D}$ geometry by the solid line in Fig. 7a, centered at $x=0$ and with a half-width equal to unity. The current flows out of the image plane towards the reader. Its volume current density is $j_{0}=1$, with a density across the sheet uniformly distributed. Antiparallel magnetic fields are located on both sides of the plane $x=0$ in accordance with Maxwell equation $\boldsymbol{\nabla} \times \boldsymbol{B}=\frac{4 \pi}{c} \boldsymbol{j}$. The neutral current sheet arises because the magnetic field vanishes at its center $(x=0)$. If the current sheet had zero width, the magnetic field would have a discontinuity at $x=0$, but we prefer to discuss here a more realistic case of a distributed current.

In quasi-magnetostatic situations (considered in this paper), the pressure balance condition in the direction perpendicular to the current sheet (i.e., along the $X$-axis) is fulfilled: $p+B^{2} / 8 \pi=$ const. If we do not consider changes of temperature in the region of interest, then the plasma density is enhanced in the neutral current sheet, thus forming a plasma sheet concentrated around the neutral current sheet because of a lower magnetic pressure inside of it.

The plasma sheet around the neutral current sheet represents the main plasma sheet in our model (the main part of the streamer belt). The secondary plasma sheet cannot be represented as a neutral sheet because the magnetic field would have to change its sign and this is not possible in the required geometry (see Fig. 2). To illustrate the possibility of forming the second plasma sheet, we again use the 1D calculation in a simplified plane-parallel geometry (see Fig. 7). Along with the main current sheet at $x=0$, two secondary current sheets of half-width $\Delta x=0.5$ (all parameters hereafter are
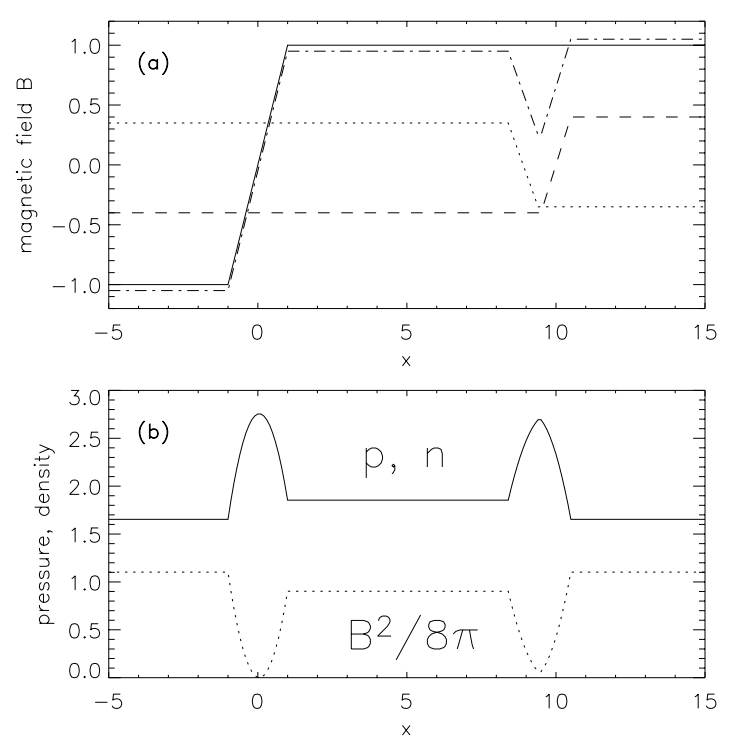

Fig. 7. Results of a simple 1D calculation illustrating a possible configuration of current sheets leading to a double plasma sheet. The magnetic field strength and the magnetic pressure, normalized to the corresponding quantities provided by the single main neutral current sheet, are plotted along the direction perpendicular to the neutral current sheet ( $x$ coordinates). a) Magnetic fields created by the main neutral current sheet centered at $x=0$ (solid line), and secondary current sheets centered at $x=8.9$ and at $x=10$ with opposite currents (dotted and dashed lines). The resulting magnetic field distribution is shown by the dash-dotted line. b) The magnetic pressure $B^{2} / 8 \pi$ (dotted line), the plasma pressure $p$, and the particle number density $n$ distributions (both represented by the solid line) at the assumed uniform temperature distribution $T=1$.

normalized to those of the main current sheet) are now centered at $x=8.9$ and $x=10$. They carry current densities $j_{1}=-0.7$ and $j_{2}=0.8$ (in opposite directions). If these current sheets were isolated, they would provide magnetic fields as shown by the dotted and dashed lines in Fig. 7a, i.e., they would be neutral. However, in the presence of sufficiently strong background field (produced by the main current sheet at $x=0$ ) they become non-neutral and the resulting distribution of the total magnetic field is shown by the dash-dotted line in Fig. 7a.

The current densities of the two secondary sheets are chosen in such a way that the resulting magnetic fields nearly cancel each other outside of them (we did not opt for a complete cancellation to keep the calculations more general). The resulting magnetic pressure distribution is shown in Fig. $7 \mathrm{~b}$ by the dotted line. Assuming a plasma parameter $\beta=\frac{p}{B^{2} / 8 \pi}=1.5$ (a reasonable value in the outer corona where the solar wind flow dominates over the magnetic field), the plasma pressure $p$ is distributed as shown by the solid line in Fig. 7b. Assuming the uniform temperature $T=1$ over all the region in consideration, the particle number density $n$ distribution has the same shape as the plasma pressure and is shown by the same solid line in Fig. 7b. The two peaks on this graph represent the two plasma sheets required by our model of the streamer belt. The first one at $x=0$ is centered around the neutral current sheet, 
the second one at $x=9.5$ is located between two non-neutral current sheets.

The simple proof-of-the-principle calculation described in this section does not attempt to represent all the aspects of the physics in coronal streamers. It is rather an example illustrating that the plasma density can be increased outside a neutral current sheet, when other current sheets are present. It is difficult to create a realistic model because of our poor knowledge of the $3 \mathrm{D}$ distribution of plasma and magnetic field parameters in the solar corona.

Figure $2 \mathrm{c}$ represents a sketch showing how secondary current sheets can be placed in order to produce the second plasma sheet. The main plasma sheet is centered around the neutral sheet carrying currents $I$ and $I_{0}$. The secondary plasma sheet is located between the currents $I_{1}$ and $I_{2}$. If the current densities are distributed according to the $1 \mathrm{D}$ proof-of-the-principle model described above, the secondary plasma sheet is confined between the currents $I_{1}$ and $I_{2}$. In Fig. 2c, the secondary current sheets represent ramifications of the main current sheet, so that the condition $I=I_{0}+I_{2}-I_{1}$ is satisfied.

Of course, other configurations of a ramification can also be proposed. The exact configuration is not too important for our 3D model because the brightest structures in the synoptic maps are created by the culmination of the secondary plasma sheet (which is nearly parallel to the equator and thus is seen edgeon). This part is reasonably well approximated by the planeparallel geometry described above and shown in Fig. 7. The instability (if any) of such a configuration of currents may represent the evolution of current sheets and their ramifications. The investigation of this process is beyond the scope of this paper. We mentioned earlier (Sect. 3) that the double plasma sheet configuration lifetime is less than a solar rotation period. This may indeed be due to the instability of the current configuration.

We note that multiple current sheets are not exotic features in solar and heliospheric physics. In situ solar wind measurements reveal the existence of numerous magnetic field discontinuities (i.e. current sheets) of different types. In particular, these discontinuities are often situated near sector boundaries, i.e. the Heliospheric Current Sheet (HCS) (see e.g. Burlaga 1968; Burlaga et al. 1977; Klein \& Burlaga 1980; Behannon et al. 1981; Mariani \& Neubauer 1990; Crooker et al. 1993; Smith 2001). About $80 \%$ of them are tangential discontinuities (Mariani \& Neubauer 1990). Multiple HCS crossings have been interpreted by Crooker et al. (1993) as a passage through multiple current sheets stemming from multiple helmet streamer arcades or directly from the solar surface. Multiple current sheets in the corona have been envisioned not only in sketches (Crooker et al. 1993), but also in MHD models (Wiegelmann et al. 1998, 2000), in particular as triple current sheets. LASCO C1 observations indicate that these three current sheets can be indeed present (Schwenn et al. 1997), but seem to merge at some radial distance to form one single HCS. Our secondary current sheets should extend high in the corona as the corresponding fine structures are present in LASCOC2 images up to at least 5 solar radii. Koutchmy \& Livshits (1992) suggested that multiple (ramified) current sheets exist in the streamer stalk to provide the closure of the main (azimuthal) current in the same manner as the cross-tail current of the Earth's magnetosphere is closed by the currents on the magnetopause.

We also point out the similarity of the secondary plasma sheet structure presented in Fig. 7 to the so-called magnetic holes (or depressions) in the solar wind (see e.g. Fitzenreiter \& Burlaga 1978; Zurbuchen et al. 2001). A magnetic hole represents a sudden dropout of the interplanetary magnetic field observed in situ. As these structures are pressure-balanced, they have increased plasma pressures and therefore often exhibit enhanced densities. The magnetic field and electric current configurations of a magnetic hole shown by Fitzenreiter \& Burlaga (1978) in their Fig. 2 is similar to the pair of secondary current sheets in our Fig. 7. Although the magnetic holes usually appear on smaller scales (Fitzenreiter \& Burlaga 1978), they can also last for several hours, see Zurbuchen et al. (2001) and references therein. So, planar magnetic holes can closely resemble our secondary plasma sheet.

It is difficult to conceive whether non-neutral current sheets can be traced in photospheric magnetograms. In principle, the method of the potential extrapolation of the photospheric field can be improved in a way to have several current sheets beyond the source surface, but this will strongly complicate the mathematical formalism. Non-neutral current sheets will appear when the background field (produced by the main neutral current sheet) is strong enough in comparison with the fields produced by secondary current sheets. Note that the total magnetic field distribution shown in Fig. 7a by the dash-dotted line is very close to the one given by the single neutral current sheet (solid line) everywhere except in a small region in between the secondary current sheets.

Regarding the CR 1913, we saw that the inspection of LASCO and MDI synoptic maps together with LASCO and EIT images allowed us to identify the "photospheric neutral line-loop system-streamer" combinations anticipated from the model of Pneuman \& Kopp (1971), and this gives an idea of the $3 \mathrm{D}$ structure of the streamer belt as a single warped current/plasma sheet. Some of the folds of the real coronal neutral line do not appear in the potential field source surface model. They are probably smoothed out in the calculation process and the resulting modeled coronal current sheet cannot represent some streamers adequately.

Among the two interpretations of the fine structure of the streamer belt proposed in this study (additional plasma sheet folds and additional plasma sheets), the first one is topologically simpler. Thus one can expect to encounter additional folds more frequently than additional plasma sheets, especially during the minimum of solar activity. At its maximum, the photospheric neutral line is more complex, and multiple coronal neutral sheets may appear. They can correspond for instance to the polar crown filaments observed in both hemispheres.

As we noted in the Introduction, an alternative explanation of the fine features in the streamer belt involves the existence of fine rays - flux tubes with increased plasma density (Liewer et al. 2001). Similarly to our interpretation with additional current sheets, the full 3D modeling of these rays has not yet been performed. The existence of the fine structures of the streamer belt requires that modern MHD models of the solar corona have 
to be modified in a way to explain them. With the current state of MHD models and the present constraint of imaging the solar corona from only one vantage point, it may be difficult to discriminate between the proposed interpretations of the streamer belt fine structure. However we note that Liewer et al. (2001) used the potential field source surface model, which, as we have demonstrated in Sect. 3, can be misleading.

\section{Conclusions}

In this paper we have attempted to improve the understanding of the 3D structure of the streamer belt of the Sun, as evidenced by the LASCO synoptic maps. We confirm previous results (e.g. Wang et al. 2000) that the large-scale features can be well explained by a single current/plasma sheet, with its location inferred from the coronal neutral line calculated within the framework of the potential field source surface model. However we found that this model is unable to account for the observed fine features seen on the LASCO synoptic maps. Two independent effects are proposed to explain these fine features.

The first effect involves additional streamers which can correspond to additional folds of the coronal neutral line. These folds are absent in the potential field source surface model, but correspond well to the folds of the underlying photospheric neutral line. The potential field source surface model is therefore not adequate to describe the fine structure of the streamer belt, even during periods of low solar activity.

The second effect is based on ramification of the main plasma sheet. In the proposed configuration of a triple current sheet/double plasma sheet, representing one of possible situations, the second plasma sheet is situated between the two secondary current sheets bearing oppositely directed currents. The secondary plasma sheet has enhanced density due to the pressure balance in the transverse direction. It still remains to be proven if such a configuration exists in the solar corona.

Our results do not exclude the existence of density inhomogeneities in the azimuthal direction of the streamer belt, but we have shown that this is not often a necessary feature. The STEREO mission (Solar - Terrestrial Relations Observatory) will hopefully clarify this issue, by looking at the streamer belt from two different vantage points in space.

Acknowledgements. This article was partially prepared when A. N. Zhukov visited the Laboratoire d'Astronomie Spatiale. We are grateful to Y.-M. Wang for making his program to calculate the magnetic field at the source surface available to us and for many helpful advices. We acknowledge comments from the unknown referee which very much helped improving the first version of this article. LASCO was built by a consortium of the Naval Research Laboratory, USA, the Laboratoire d'Astronomie Spatiale in Marseille, France, the Max Planck Institut für Aeronomie in Lindau, Germany, and the School of Physics and Astronomy, University of Birmingham, UK. EIT data have been used courtesy of the SOHO/EIT consortium. SOHO is a project of international cooperation between ESA and NASA. NSO/Kitt Peak data used here are produced cooperatively by NSF/NOAO, NASA/GSFC, and NOAA/SEL.

\section{References}

Badalyan, O. G., Livshits, M. A., \& Sýkora, J. 1997, Sol. Phys., 173, 67

Behannon, K. W., Neubauer, F. M., \& Barnstorf, H. 1981, J. Geophys. Res., 86, 3273

Brueckner, G. E., Howard, R. A., Koomen, M. J., et al. 1995, Sol. Phys., 162, 357

Burlaga, L. F. 1968, Sol. Phys., 4, 67

Burlaga, L. F., Lemaire, J. F., \& Turner, J. M. 1977, J. Geophys. Res., 82, 3191

Crooker, N. U., Siscoe, G. L., Shodhan, S., et al. 1993, J. Geophys. Res., 98, 9371

Delaboudinière, J.-P., Artzner, G. E., Brunaud, J., et al. 1995, Sol. Phys., 162, 291

Eselevich, V. G., \& Eselevich, M. V. 1999, Sol. Phys., 188, 299

Fitzenreiter, R. J., \& Burlaga, L. F. 1978, J. Geophys. Res., 83, 5579

Howard, R. A., \& Koomen, M. J. 1974, Sol. Phys., 37, 469

Klein, L., \& Burlaga, L. F. 1980, J. Geophys. Res., 85, 2269

Koomen, M. J., Howard, R. A., \& Michels, D. J. 1998, Sol. Phys., 180, 247

Koutchmy, O., \& Koutchmy, S. 1989, in High Spatial Resolution Solar Observations, Proc. of the 10th Sacramento Peak Summer Workshop, ed. O. von der Lühe, 217

Koutchmy, S. 1972, Sol. Phys., 24, 373

Koutchmy, S., \& Livshits, M. 1992, Space Sci. Rev., 61, 393

Lamy, P., Llebaria, A., \& Quemerais, E. 2002, Advances in Space Research, 29, 373

Liewer, P. C., Hall, J. R., de Jong, M., et al. 2001, J. Geophys. Res., 106,15903

Linker, J. A., Mikić, Z., Biesecker, D. A., et al. 1999, J. Geophys. Res., 104, 9809

Mariani, F., \& Neubauer, F. M. 1990, in Physics of the Inner Heliosphere I, ed. R. Schwenn, \& E. Marsch (Berlin: Springer-Verlag), 183

Molodensky, M. M., Starkova, L. I., Koutchmy, S. L., \& Ershov, A. V. 1996, in Solar Drivers of the Interplanetary and Terrestrial Disturbances, ASP Conf. Ser., 95, 385

Pneuman, G. W., \& Kopp, R. A. 1971, Sol. Phys., 18, 258

Saez, F., Llebaria, A., Lamy, P., \& Vibert, D. 2005, A\&A, submitted

Schatten, K. H. 1971, Cosmic Electrodynamics, 2, 232

Schwenn, R., Inhester, B., Plunkett, S. P., et al. 1997, Sol. Phys., 175, 667

Smith, E. J. 2001, J. Geophys. Res., 15819

Vedenov, A. A., Koutvitsky, V. A., Koutchmy, S., Molodensky, M. M., \& Oraevsky, V. N. 2000, Astronomy Reports, 44, 112

Vibert, D., Llebaria, A., Netter, T., Balard, L., \& Lamy, P. 1997, in Astronomical Data Analysis Software and Systems VI, ASP Conf. Ser., 125, 230

Vsekhsvyatsky, S. K., Nikolsky, G. M., Ivanchuk, V. I., et al. 1965, The Solar Corona and Corpuscular Emission in the Interplanetary Space (Kiev: Kiev University Press), in Russian

Wang, Y.-M., Sheeley, N. R., Howard, R. A., et al. 1997, ApJ, 485, 875

Wang, Y.-M., Sheeley, N. R., \& Rich, N. B. 2000, Geophys. Res. Lett., 27, 149

Wiegelmann, T., Schindler, K., \& Neukirch, T. 1998, Sol. Phys., 180, 439

Wiegelmann, T., Schindler, K., \& Neukirch, T. 2000, Sol. Phys., 191, 391

Zurbuchen, T. H., Hefti, S., Fisk, L. A., et al. 2001, J. Geophys. Res., 16001 